\title{
Prevalence and antibiotic resistance patterns of Proteus mirabilis isolated from catheter-associated urinary tract infection.
}

\author{
Fathy M. Serry, Eman M. El-Masry, Refaat A. Sadek, Marian M. Girgis \\ Department of Microbiology and Immunology-Faculty of Pharmacy-Zagazig \\ University- Zagazig Egypt
}

\begin{abstract}
The aim of this study was to determine the frequency of the bacterial agents that cause urinary tract infection in catheterized patients at Zagazig University Hospital and to assess the antimicrobial resistance pattern of Proteus mirabilis isolates against various antimicrobial agents used for treating urinary tract infections in the spate of its recorded increasing resistance patterns.Urine specimens were obtained from catherized patients suffering from a urinary tract infection (UTI). The different isolates obtained were identified by conventional microbiological methods. The antibiotic susceptibility profile of Proteus mirabilis isolates was determined by agar dilution method. Patient's medical records showed significant increase in incidence of catheter associated urinary tract infection (CA-UTI) upon prolonged catheterization, among immunocompromised patients and patients with concomitant chronic diseases, elderly patients and female gender.

The frequency of the obtained isolates was Eschersheia coli (33\%), Klebsiella pneumoniae (14\%), Enterococcus faecalis (13\%), Proteus species (10.2\%), Pseudomonas aeruginosa (10\%), Staphylococcus aureus (10\%) and unidentified gram positive cocci (9.8\%). 90\% of the Proteus isolates were Proteus mirabilis. The resistance of $P$. mirabilis isolates were highest against Nalidixic acid, Ampicillin/Sulbactam, Amoxicillin/clavulanic acid, ceftazidime and cefuroxime (95.5\%-57.8\%). Lower resistance rates of P. mirabilis were found against amikacin, levoflxacin, ciporofloxacin and gentamicin (2.2 \% $-17.8 \%)$. While P.mirabilis isolates were $100 \%$ sensitive to Imipenem. In conclusion, Imipenem is recommended to be used as an empirical treatment of CA-UTI caused by P. mirabilis.
\end{abstract}

\section{INTRODUCTION}

Urinary tract infections are the most common infections after upper respiratory tract infections (Hryniewicz et al., 2001) and they are the most prevalent type of nosocomial infections accounting for $25 \%$ to $40 \%$ of nosocomial infections (Bagshaw and Laupland, 2006). Catheter associated urinary tract infections (CA-UTI) are the most common type of nosocomial infections, accounting for $80 \%$ of all nosocomial UTIs (Hartstein , 1981). Bacteria are the major causative organisms for UTIs and are responsible for more than $95 \%$ of UTI cases (Bonadio et al., 2001). Proteus mirabilis is the third most common cause of complicated UTI (12\%) and the second most common cause of catheter-associated bacteriuria in long term catheterized patients $(15 \%)$ (Warren, 1996). It was observed that $P$. mirabilis has the greatest ability to attach to catheters out of all gramnegative organisms (Roberts et al.,1990). The ability of P. mirabilis to colonize the surfaces of catheters and urinary tract is believed to be aided by a characteristic known as swarming differentiation and migration (Allison et al.,1994). Furthermore, the expression of other virulence factors of Proteus including urease and protease is coordinately upregulated during swarming (Rather, 2005). Antimicrobial resistance offered by different uropathogens is one of the barricades that might hinder a 
successful treatment as antimicrobial resistance pattern varies with time ( Dyer,1998). Lack of appropriate laboratory facilities to carry out comprehensive antimicrobial sensitivity patterns leads to wrong empirical drug selections with attendant treatment failures and propagation of resistant bacteria (Noreddin et al., 2011).

This study was set up to investigate the prevalence and antibiotic susceptibility patterns of uropathogenic Proteus isolated from catheterized patients at Zagazig University Hospital so as to offer a guide to clinicians for proper antibiotic selection for empirical treatment.

\section{MATERIALS AND METHODS Media and chemicals}

MacConkey agar, Cysteine lactose electrolyte deficient agar (CLED), Nutrient agar, Mannitol salt agar, Eosin-methylene blue (EMB) agar, Mueller Hinton agar were obtained in dehydrated form from Oxoid, Hampshire, England (Hampshire, UK). Triple sugar iron (TSI) agar, Simmon's citrate agar and Bile esculin agar was purchased from Lab M Limited (Lancashire, United Kingdom.). The other culture media were prepared from their components according to Koneman et al. (1997).

Antimicrobial agents were of pharmaceutical grades. A total of thirteen antimicrobial agents were used in the study.Azetronam was obtained from Bristol-Myers Squib, Egypt. Amoxicillin /clavulinic acid were obtained from Sedico pharmaceutical company,Egypt.

Ceftriaxone, cefuroxime and imipenem was obtained from Glaxo Smith Kline, Egypt. Ampicillin/sulbactam and cefoperazone was obtained from Pfizer, Egypt. Ciprofloxacin, levofloxacin, cefotaxime, amikacin and gentamicin were obtained from
Egyptian Pharmaceutical Industries Company (EPICO), Egypt. Ceftazidime was obtained from Smith Kline Beecham, Egypt. Nalidixic was obtained from Memphis Chemical company, Egypt. Nitrofurantoin was obtained from Mepaco-Medifood, Egypt.

Isolation and idetification of the isolates

A total of 520 urine specimens were collected from catheterized patients with urinary tract infections comprising 203 males and 317 females aged between 4 years to 80 years were assessed between the period from October 2009 to September 2010. The samples were collected from patients admitted to Zagazig University Hospital. All patients have been catheterized minimum for 4 days and maximum for 30 days. All specimens were aseptically collected and transported immediately to the microbiological laboratory, where they were processed. The specimens were cultured on Blood agar, CLED agar using $1 \mu \mathrm{l}$ calibrated loop and incubated aerobically at $37^{\circ} \mathrm{C}$ for $24 \mathrm{~h}$ to detect colony forming unit, count more than $10^{5} \mathrm{cfu} / \mathrm{ml}$ was considered significant. The isolates were identified by morphological, Gram stain and biochemical reactions according to Koneman et al. (1997).

\section{Determination of minimum inhibitory concentration (MIC) by the agar dilution method}

Minimum inhibitory concentrations (MIC $\mu \mathrm{g} / \mathrm{ml}$ ) of the selected antimicrobial agents against Proteus mirabilis isolates were determined by agar dilution method according to Clinical Laboratory and Standards Institute (CLSI, 2010).

\section{Preparation of the inoculum}

From a pure culture, at least 3-4 colonies were picked. Resolve totally in $4-5 \mathrm{ml}$ saline, mix and adjust 
turbidity to 0.5 McFarland standard. The bacterial suspension then diluted 10 -fold to yield the final inoculums suspension of $10^{7} \mathrm{CFU} / \mathrm{ml}$. The bacterial suspension should be used for inoculation within 15 minutes.

\section{Preparation of agar dilution plates}

The appropriate dilutions of antimicrobial solutions were prepared and added to molten Mueller-Hinton agar $(3 \%$ agar to minimize the swarming) at 45 to $50^{\circ} \mathrm{C}$, mixed and poured into petri dishes.

\section{Inoculating agar plates}

A standardized micropipette was used to deliver $1 \mu \mathrm{l}$ of the suspension to the surface of Mueller-Hinton agar containing antibiotic dilutions and the control plates (without the addition of the antimicrobial agent), so that the final inoculum on the agar contains approximately $10^{4} \mathrm{cfu} / \mathrm{spot}$. The inoculated agar plates were allowed to stand at room temperature until the liquid was absorbed into the agar. The plates were inverted and incubated at $35-37^{\circ} \mathrm{C}$ for $16-20$ hours, and examined to determine the MIC. The results were recorded and interpreted according to CLSI (2010) as susceptible (S), intermediate (I) and resistant $(\mathrm{R})$.

\section{STATISTICAL ANALYSIS}

Statistical package for social sciences (SPSS; version 10; USA) was used to analyze data. Comparison of categorical variables and percentages between groups was done by onesample $T$ test, one-way ANOVA, as appropriate. A probability of $\mathrm{P}<0.05$ was considered significant.

\section{RESULTS}

Patient's medical records were reviewed to identify the duration of catheterization and other factors that may contribute to the development of catheter associated urinary tract infection (CA-UTI). Figure (1) showed significant increase in the incidence of catheter associated urinary tract infection upon prolonged catheterization. In addition, patients' medical records (data not presented) cleared that $82 \%$ of the patients were immunocompromised and had concomitant chronic diseases such as diabetes, renal dysfunction, cancer, cardiovascular events and neurologic disorders (data not presented). Table (1) revealed the percentages of occurrence of CA-UTIs among the different age/gender groups. In females the highest percentage $(52.7 \%)$ of CAUTI cases was observed in patients who are aged $\geq 60$ years, followed by $(14.2 \%)$ in patients aged 50-59 years. However, the highest percentage $(42.9 \%)$ of CA-UTI in males was observed in patients who are aged $\geq 60$ years, followed by (15.7\%) among patients aged 40-49 years. Furthermore, $9.6 \%$ of CA-UTI were children under 10 years. While there was no significant difference between males and females at the age group 4-9 years $(P>0.05)$. In addition, our results suggested that the occurrence of CA-UTIs in females is significantly higher than that of male $(P<0.05)$. There was significant difference between the total number of male and female patients in the incidence of occurrence of CA-UTIs (Table 1 \& Figure 2).

Out of 520 urine specimens collected from catheterized patients with urinary tract infections in Zagazig University Hospital from Department of Urology and Intensive Care Units from October 2009 to September 2010. 490 specimens (94\%) gave positive bacterial growth with a viable count $>10^{5} \mathrm{cfu} / \mathrm{ml}$. The isolates recovered were identified and results were presented in table (2). Escherichia coli was the most frequently isolated pathogen (33\%), followed by Klebsiella pneumonia (14\%), 
Enterococcus faecalis (13\%), Proteus spp (10.2\%), Pseudomonas aeruginosa (10\%), Staphylococcus aureus (10\%) and unidentified Gram positive cocci
(9.8\%). In addition, out of the fifty Proteus isolates, 45 were identified as Proteus mirabilis $(90 \%)$, while 5 were Proteus penneri (10\%).

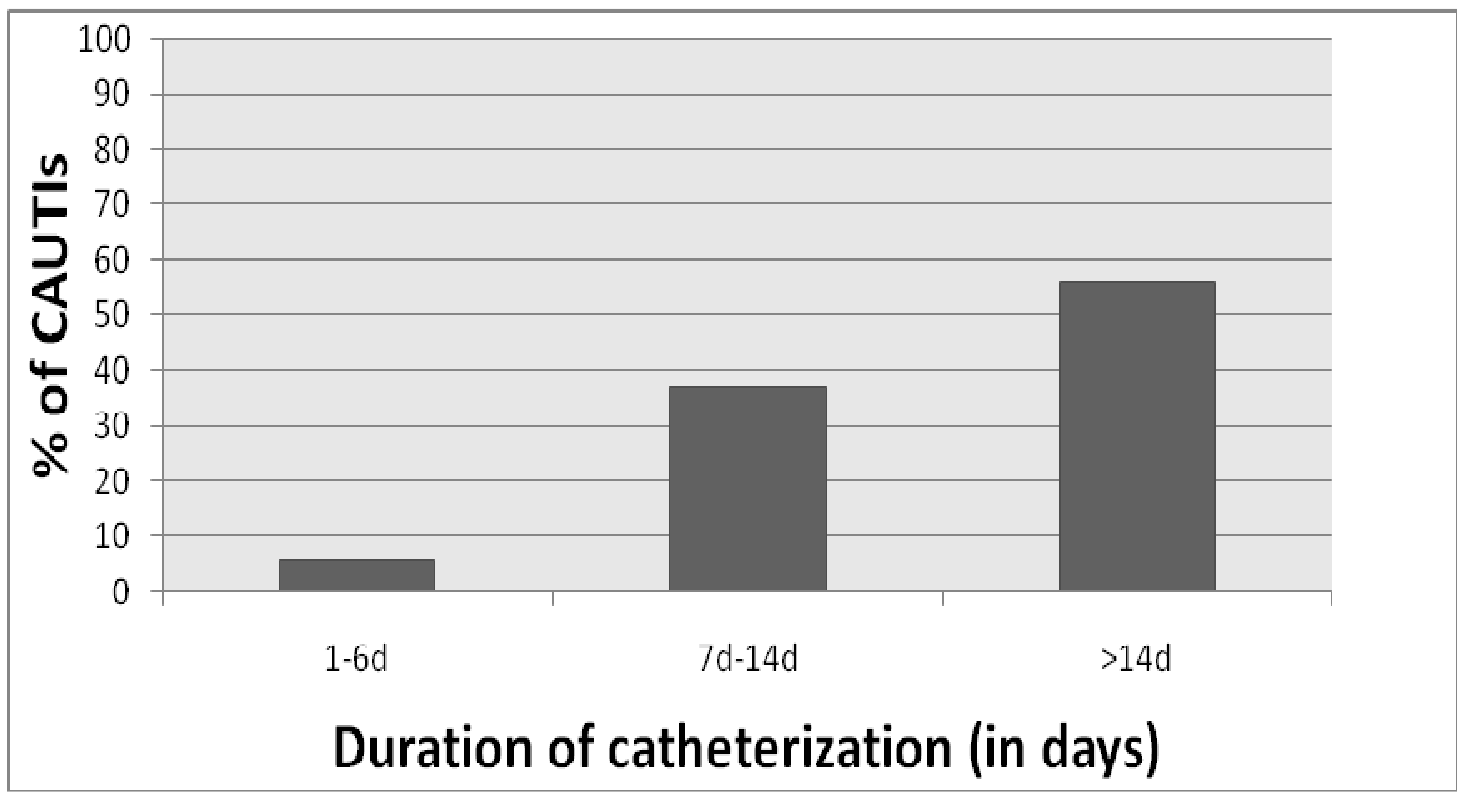

Figure 1. Correlation between duration of catheterization and occurrence of Catheter associated urinary tract infections.

Table 1. Age and gender distribution of catheter associated UTIs.

\begin{tabular}{lcccccc}
\hline Age (years) & \multicolumn{2}{c}{ Male } & \multicolumn{2}{c}{ Female } & \multicolumn{2}{c}{ Total } \\
\cline { 2 - 7 } $4-9$ & No. & $\%$ & No. & $\%$ & No. & $\%$ \\
\cline { 2 - 7 } $10-19$ & 20 & 9.8 & 30 & 9.5 & 50 & 9.6 \\
$20-29$ & 15 & 7.4 & $20^{*}$ & 6.3 & 35 & 6.7 \\
$30-39$ & 12 & 6 & $14^{*}$ & 4.4 & 26 & 5 \\
$40-49$ & 11 & 5.4 & $20^{*}$ & 6.3 & 31 & 6 \\
$50-59$ & 32 & 15.7 & $21^{*}$ & 6.6 & 53 & 10.2 \\
$\geq 60$ & 26 & 12.8 & $45^{*}$ & 14.2 & 71 & 13.7 \\
Total & 87 & 42.9 & $167^{*}$ & 52.7 & 254 & 48.8 \\
\hline
\end{tabular}

* Significantly higher $(\mathrm{P}<0.05)$ by one-sample $T$ test

Table 2. Frequency of the isolated pathogens from CA-UTIs.

\begin{tabular}{lcc}
\hline Bacterial isolate & No. & $\%$ \\
Escherichia coli & 162 & 33 \\
Klebsiella pneumonia & 68 & 14 \\
Enterococcus faecalis & 64 & 13 \\
Proteus spp & 50 & 10.2 \\
Pseudomonas aeruginosa & 49 & 10 \\
Staphylococcus aureus & 49 & 10 \\
Unidentified Gram positive cocci & 48 & 9.8 \\
\hline
\end{tabular}




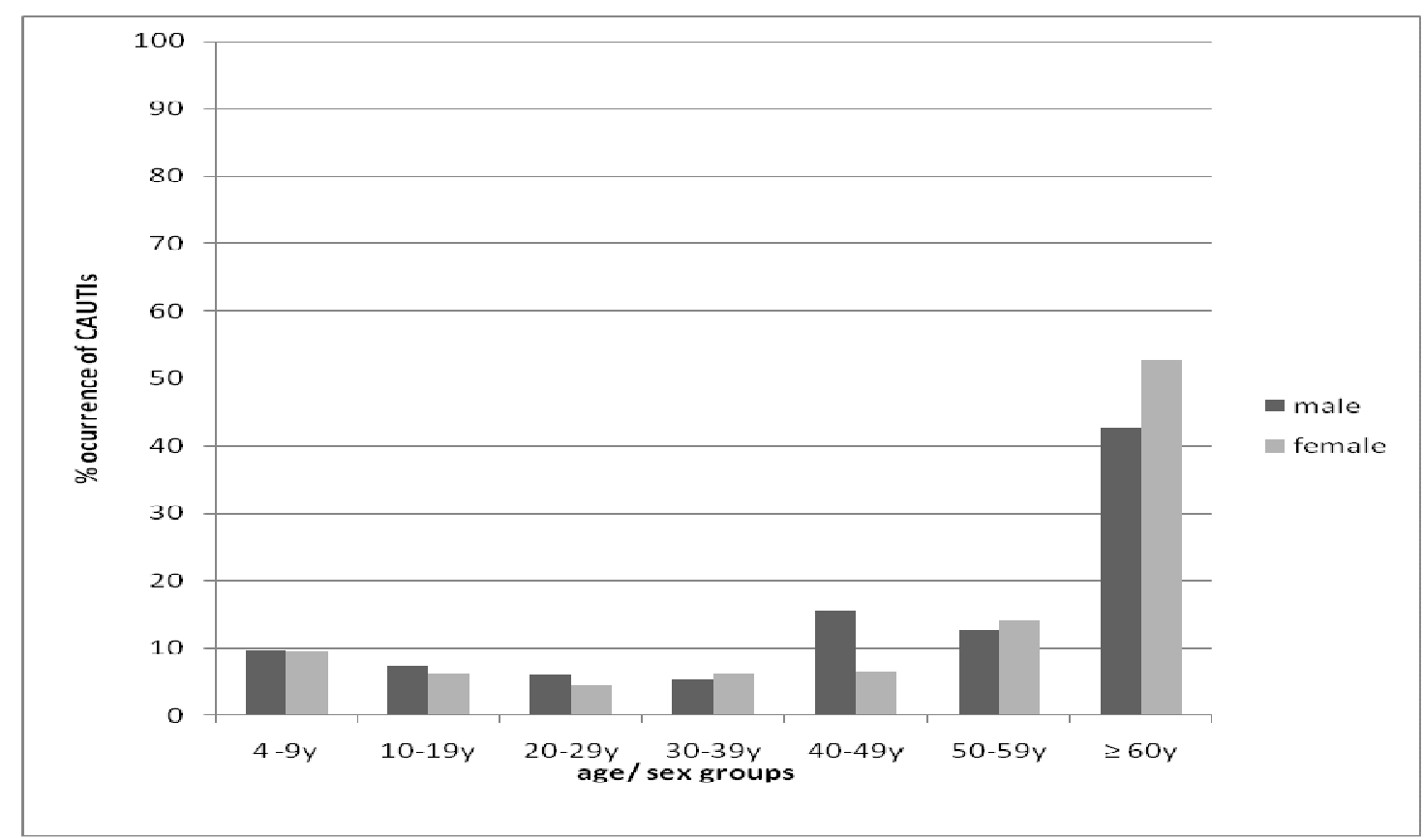

Figure 2. Correlation between age/gender and occurrence of CA-UTIs.

Minimum Inhibitory Concentration (MIC) of antimicrobial agents

The percentage of resistance of Proteus mirabilis to different antimicrobial agents used is presented in (Table 3). $\mathrm{MIC}_{50}$ and $\mathrm{MIC}_{90}$ of various antimicrobial chemotherapeutic agents against
Proteus mirabilis isolated from the indwelling catheters are presented in (Table 4) in terms of $\mathrm{MIC}_{50}$ (MIC at which $50 \%$ of the isolates tested were inhibited), $\mathrm{MIC}_{90}$ (MIC at which $90 \%$ of the isolates tested were inhibited), and the range of MICs

Table 3. The percentage of resistance of P.mirabilis against various antimicrobial chemotherapeutic agents

\begin{tabular}{lc}
\hline \multicolumn{1}{c}{ Antimicrobial agent } & Resistance rates of P.mirabilis \\
\hline Imipenem & $0 \%$ \\
Amikacin & $2.2 \%$ \\
Gentamicin & $17.8 \%$ \\
Aztreonam & $33.3 \%$ \\
Cefotaxime & $40 \%$ \\
Cetazidime & $60 \%$ \\
Cefuroxime & $57.8 \%$ \\
Ciprofloxacin & $8.8 \%$ \\
Levofloxacin & $4.5 \%$ \\
Amoxicillin/Clavulinic acid & $64.4 \%$ \\
Ampicillin/Salbactam & $75.5 \%$ \\
Nalidixic & $95.5 \%$ \\
Nitrofurantoin & $48.8 \%$ \\
\hline
\end{tabular}


Table 4. $\mathrm{MIC}_{50}$ and $\mathrm{MIC}_{90}$ of various antimicrobial chemotherapeutic agents' against P. mirabilis

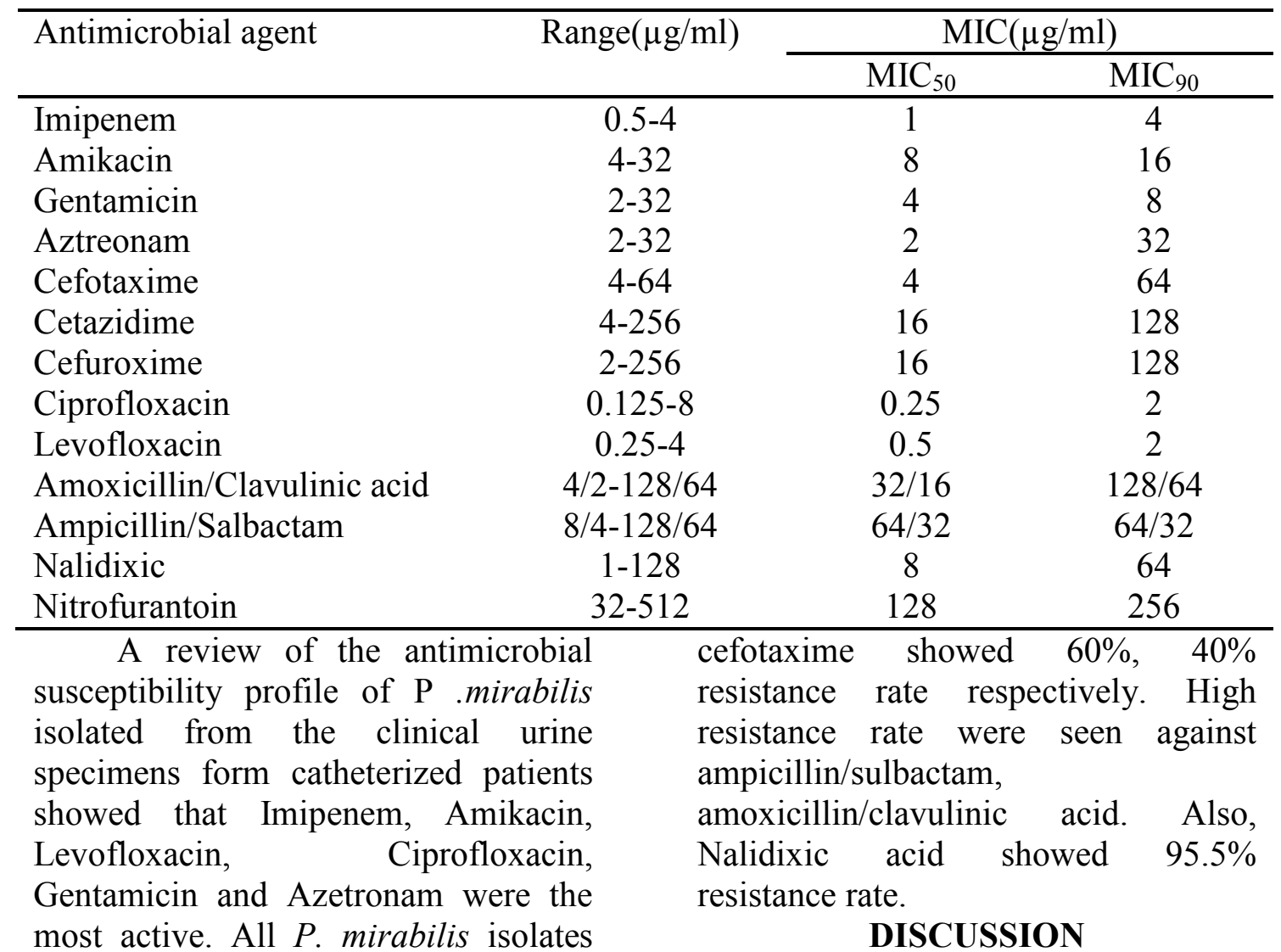
were sensitive to Imipenem with $\mathrm{MIC}_{50}$ and $\mathrm{MIC}_{90}$ of $1 \mu \mathrm{g} / \mathrm{ml}, 4 \mu \mathrm{g} / \mathrm{ml}$, respectively. Also, Aminoglycosides were highly active on $P$. mirabilis. Amikacin showed greater activity than Gentamicin as the resistance against Amikacin was $2.2 \%$. On the other hand, resistance against Gentamicin was $17.8 \%$. Furthermore, Quinolones (Levofloxacin and Ciprofloxacin) showed high activity on $P$. mirabilis isolates. Levofloxacin had an $\mathrm{MIC}_{50}$ and $\mathrm{MIC}_{90} \quad$ of $\quad 0.5 \mu \mathrm{g} / \mathrm{ml}, \quad 2 \mu \mathrm{g} / \mathrm{ml}$ respectively, while Ciprofloxacin had an $\mathrm{MIC}_{50 \text { and }} \mathrm{MIC}_{90} 0.25 \mu \mathrm{g} / \mathrm{ml}, 2 \mu \mathrm{g} / \mathrm{ml}$ respectively. $P$. mirabilis isolates showed 33.3\% resistance to Aztreonam which is representative to monbactams. Nitrofurantoin was active on about half the $P$. mirabilis isolates, as the resistance rate against it was $48.8 \%$. Cephalosporins as cefuroxime (second generation) had a resistance rate of $57.8 \%$, while third generation cephalosporins as ceftazidime and

Urinary tract infection (UTI) remains a worldwide therapeutic problem. Early diagnosis and prompt antimicrobial treatment are required to minimize its complications. The risk of developing a urinary tract infection increases significantly with the use of indwelling catheters .CA-UTIs can lead to many complications as cystitis, pyelonephritis, gram-negative bacteremia, prostatitis, epididymitis, and orchitis in males and, less commonly, endocarditis, vertebral osteomyelitis, septic arthritis, endophthalmitis, and meningitis in all patients (Foxman, 2003).

In the present study done in Zagazig university hospital, Escherichia coli (33\%) was the most prevalent cause of CA-UTIs followed by Klebsiella pneumonia (14\%), Enterococcus faecalis (13\%), Proteus spp (10.2\%), Pseudomonas aeruginosa (10\%), Staphylococcus aureus (10\%) 
and Coagulase negative Staphylococci (9.8\%). In a study by Warren (1996), Proteus mirabilis was the second most common cause of catheter associated bacteriemia in a group of long term catheterized patients.

Proteus mirabilis was found to be the most common Proteus species isolated from UTIs (Orrett, 1999). Other Proteus spp were found to be implicated in UTIs as Proteus vulgaris and Proteus penneri (Penner, 1992). This is similar to the results reported in our study as $90 \%$ out of the fifty Proteus isolates were Proteus mirabilis and $10 \%$ were Proteus penneri.

The results show that the number of CA-UI is higher with long catheterization. This is confident with results of other study by Hass (1982), who found that the risk of getting a CA-UTI increases at $5 \%$ per day of indwell time. Older age shows higher occurrence of CA-UTIs .In current study in both sexes the incidence of CA-UTIs was the highest in patient over 60 years. Senior (1987) reported that urinary tract infections with Proteus, were frequently only in patients of 60 years and above. Geriatrics may show higher incidence due to immuno-comprimisation. The frequency of UTI is greater in women $61 \%$ as compared to men $39 \%$ similar to results that were shown by Kashef et al. (2010). This could be owing to difference in anatomic and physical factors between genders.

Antimicrobial resistance profile of Proteus isolates revealed high resistance rates to six of the most frequently used antibiotics. $P$. mirabilis showed resistance rates of $95.5 \%$ to Nalidixic acid, $75.5 \%$ to Ampicillin/Sulbactam, $\quad 64.4 \%$ to Amoxicillin/Clavulinic acid, $60 \%$ to Ceftazidime, $57.8 \%$ to Cefuroxime and $48.8 \%$ to Nitrofurantoin.
In the current study, $P$. mirabilis showed $48.8 \%$ resistance to nitrofurnatoin. In a study conducted in hospitals in Qassim province at Saudi Arabia the resistance rates of Proteus spp. to nitrofurantion was $81 \%$ (Alzohairy \& Khadri, 2011). Therefore, the current study revealed that resistance of $P$. mirabilis to Nitrofurantin has increased greatly than that which was previously reported in a university hospital in Nigeria where the resistence rate was about 25\% (Jombo et al., 2012).

Moreover, resistance rates of $P$. mirabilis to Amoxicillin/ Clavulainic acid was $64.4 \%$ which is higher than that reported by Stratchounski et al. (1998), as they showed that the resistance rate was $20 \%$. In another study in Nigeria by Jombo et al (2012) the resistance of $P$.mirabilis isolates was $62 \%$. Consequently, it is apparent that the resistance rates are increasing with time.

The resistance rates of $P$. mirabilis to the different cephalosporins used in this study were $60 \%$ to Ceftazidime, $57.8 \%$ to Cefuroxime and $40 \%$ to Cefotaxime. The current study reflects higher resistance profiles than previously reported by Stratchounski et al. (1998) who showed that the resistance rates of Proteus isolates to ceftazidime , cefotaxime were $1 \%, 20 \%$ respectively.

The resistance profile of the $P$. mirabilis to Nalidixic acid was much higher than that reported in a university hospital in Nigeria where the resistance rate was about $25 \%$ (Jombo et al., 2012).

The decrease in the susceptibility rates of Proteus isolates in the current study could be attributed to the overuse of nitrofurantoin, nalidixic, penicillins and cephalosporins in the hospital form which these isolates were collected, and to the misuse of these antibiotics. 
These antibiotics are the most used as empiric treat without performing an antibiotic sensitivity test.

The sensitivity pattern for Proteus in this study shows that Imipenem was the most effective drug for Proteus induced urinary tract infection followed by Amikacin. This is in agreement with the results of Savas et al. (2006) who found that the most effective antibiotics against Gram-negative bacteria were imipenem and meropenem. While the resistance rate of $P$. mirabilis to amikacin was $2.2 \%$.

In addition the current study showed that the resistance rate of $P$. mirabilis to fluroquinolones is higher than the study of Shigemura, (2009) who reported $100 \%$ effectiveness of Ciprofloxacin. The widespread of flouroquinolones as ciprofloxacin and levofloxacin may have accelerated the development of resistance to these agents (Livermore et al., 2002).

In conclusion, the present study had shown that Proteus mirabilis isolates from urinary tract infections were highly resistant to most of the antibiotics in common use. Prudent and judicious use of antibiotics among physicians should be emphasized while control and spread of nosocomial infections should be seriously checked to limit the spread of resistant bacteria. Furthermore, Imipenem and Amikacin may be considered for empirical treatment of Proteus mirabilis in CAUTIs if susceptibility reports are not readily available.

\section{REFERENCES}

1. Allison, C.; Emody, L.; Coleman, N.; \& Hughes, C. (1994). The role of swarm cell differentiation and multicellular migration in the uropathogenicity of Proteus mirabilis. J Infect Dis. 169:11551158.
2. Alzohairy, M.; Habeeb, K. (2011): Ferquency and Antibiotic Susceptibility Pattern of Uropathogens Isolated from Community and Hospital-Acquired Infections in Saudi Arabia-A Prospective Case Study. British Journal of Medicine \& Medical Research, 1: 45-56.

3. Bagshaw, SM; Laupland, KB (2006). Epidemiology of intensive care unit acquired urinary tract infections. Curr. Opin. Infect. Dis., 19: 67-71.

4. Bonadio, M.; Meini, M.; Spetaleri, P; Gilgi, C. (2001). Current microbiological and clinical aspects of urinary tract infections. Eur. J. Urol., 40, 439-45.

5. Clinical Laboratory Standards Institute (CLSI) (2010).

Performance Standards for Antimicrobial Susceptibility Testing; twentieth Informational Supplement M100-S20vol.30 no. 1 and Performance Standards for Antimicrobial Susceptibility Testing; twentieth Informational Supplement (June 2010 update) M100S20-U, 3: 1.

6. Dyer, IE; Sankary, IM; Dawson, JO. 1998 Antibiotic Resistance inBacterial Urinary Tract Infections, 1991 to 1997.West J. Med. 169: 265-268.

7. Foxman, B. (2003). Epidemiology of urinary tract infections: incidence, morbidity and economic costs. Dis. Mon. 49: 53-70.

8. Haas, W.; Gearinger, LS; Usner, DW; DeCory Warren, J. W., Tenney, J.H., Hoopes, J. M., Muncie, H. L. \& Anthony,W. C. (1982). A prospective microbiologic study of bacteriuria in patients with chronic indwelling urethral catheters. J. Infect. Dis. 146,719-723. 
9. Hartstein, A. I., Garber, S. B.; Ward, T. T.; Jones, S. R.; and Morthland,V. H. (1989). Nosocomial urinary tract infection: a prospective evaluation of 108 catheterized patients. Infect. Control 2:380-386.

10. Hryniewicz, K.; Szczypa, K.; Sulikowska, A.; Jankowski, K.; Betlejewska, K.; Hryniewicz, W. (2001). Antibiotic susceptibility of bacterial strains isolated from urinary tract infections in Poland. J. Antimicrob. Chemother. 47, 77380.

11. Jombo, GTA; Emanghe, UE; Amefule, EN; Damen, JG (2012).Nosocomial and community acquired uropathogenic isolates of Proteus mirabilis and antimicrobial susceptibility profiles at a university hospital in sub-saharan Africa. Asian Paicfic Journal of Tropical Disease 7-11.

12. Kashef, N.; Djavid, GE; Shahbazi, S. (2010). Antimicrobial susceptibility patterns of community-acquired uropathogens in Tehran, Iran. J. Infect. Dev. Ctries. 14: 202-206.

13. Koneman, E.W.; Allen, S.D.; and Janda, W.M. et al. (1997): Color Atlas and Textbook of diagnostic microbiology. $5^{\text {th }}$ ed. By J.B. Lippincott company, Philadelphia. New York. pp: 171-252.

14. Livermore, D.M.; James, D.; Reacher, M.;Graham, C.;Nichols T.; Stephens, P.; Johnson, A.P. and George, R.C. (2002): Trends of flouroquinolone (ciprofloxacin) resistance in Enterobacteriaceae from bacteremias, England and Wales, 1990-1999. Emerg. Infect. Dis.8:473-478.

15. Noreddin, AM; Elkhatib, WF; Cunnion, KM; Zhanel, GG. (2011): Cumulative clinical experience from over a decade of use levofloxacin in communityacquired pneumonia. Drug Healthc Patient Saf; 3: 59-68.

16. Orrett, F. A. (1999): Prevelance of Proteus species in urinary tract infections in a regional hospital in Trinidad. Chinese Med. J.; 62:438442.

17. Rather, P.N. (2005) Swarmer cell differentiation in Proteus mirabilis. Environ. Microbiol. 7, 1065-1073.

18. Roberts, J. A., E. N. Fussell, and M. B. Kaack. (1990). Bacterial adherence to urethral catheters. J. Urol. 144:264-269.

19. Savas L, Guvel S, Onlen Y, Savas N, Duran N. (2006). Nosocomial urinary tract infections: micro-organisms, antibiotic sensitivities and risk factors. West Indian Med. J., 55 (3): 188-193.

20. Senior, B.W.; Albrechtsen, M.; and Kerr, M .A. (1987): Proteus mirabilis strains of diverse type have IgA protease activity. J. Med. Microbiol. 24:175-180.

21. Shigemura K.; Arakawa S.; Tanaka K and Fujisawa M. (2009). Clinical Investigation of Isolated Bacteria from Urinary Tracts of Hospitalized Patients and their Susceptibilities to Antibiotics. J. Infect. Chemother. 15: 18-22.

22. Stratchounski, L.S; Kozlov, R.S.; Rechdko, G.K.; Stetsiouk, O.U.; and Chavrikova, E.P. (1998): Antimicrobial Resistance Patterns Among aerobic Gram-Negative Bacilli Isolated from patients in Intensive Care Units .Clin. Microbiol. Infect.9: 497-507.

23. Warren, J. W. (1996): Clinical presentations and epidemiology of urinary tract infections, In H. L. Mobley and J. W. Warren (ed.), Urinary tract infections: molecular pathogenesis and clinical management. ASM Press, Washington, DC. 


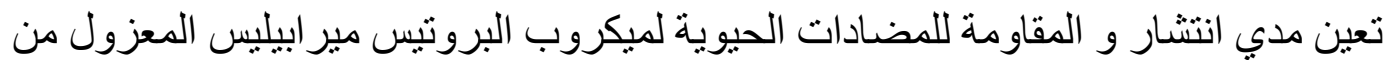

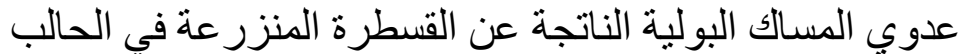

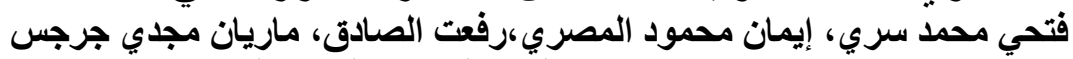

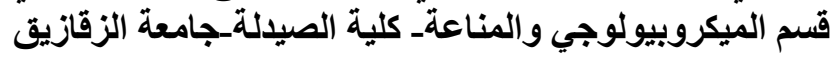

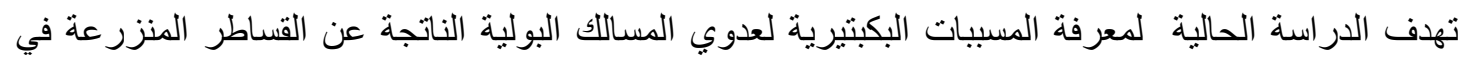

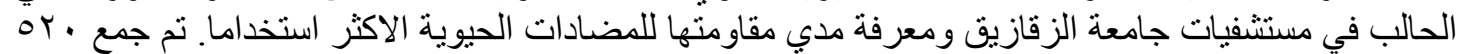

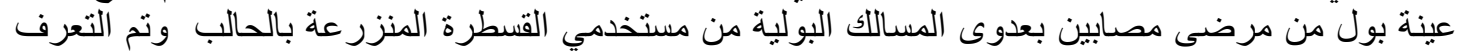

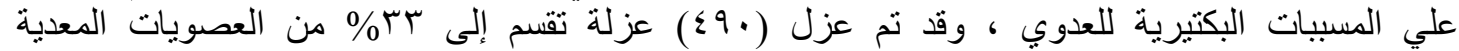

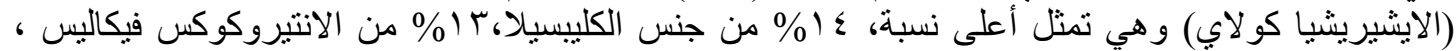

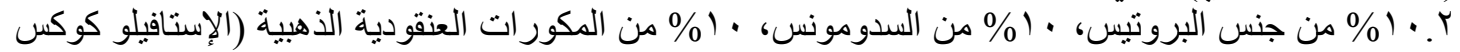

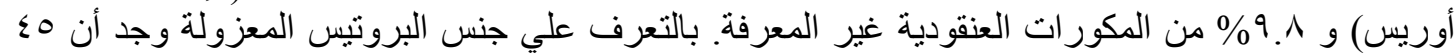

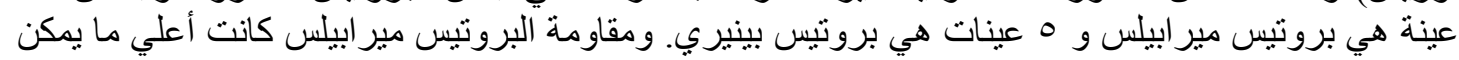

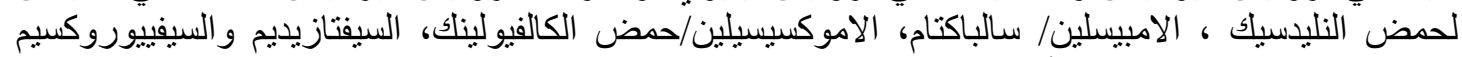

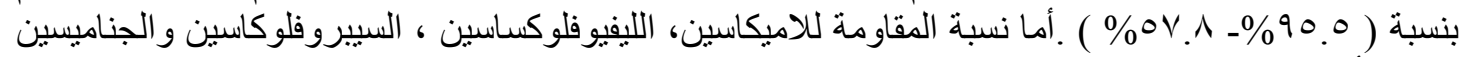

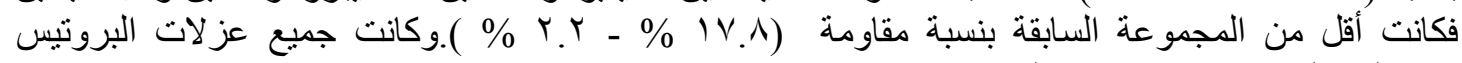

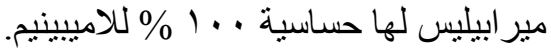

